\title{
El enfoque intersectorial en la provisión de orientación y apoyo escolar. Perspectiva de orientadores, tutores y directores
}

\section{The intersectorial approach in providing guidance and school support. The perspective of counsellors, tutors and principals}

\author{
Consuelo VÉLAZ-DE-MEDRANO, Esther LÓPEZ-MARTÍN, Eva EXPÓSITO- \\ CASAS y Ana GONZÁLEZ-BENITO \\ Universidad Nacional de Educación a Distancia
}

Recibido: Enero 2015

Evaluado: Abril 2015

Aceptado: Mayo 2015

\section{Resumen}

La provisión de orientación y apoyo educativo requiere del trabajo conjunto y la colaboración de diversos profesionales e instituciones, especialmente cuando nos encontramos ante problemas complejos que requieren un elevado grado de especialización y la combinación de conocimientos procedentes de diversas áreas. En la presente investigación se ha perseguido analizar la proximidad del sistema institucional de orientación y apoyo a la escuela en nueve Comunidades Autónomas, al enfoque intersectorial de la orientación. Para ello, se presentan los resultados de un estudio descriptivo, mediante encuesta, que permite conocer la opinión de los orientadores, tutores y directores de Educación Primaria y Educación Secundaria Obligatoria, acerca de la colaboración con los servicios públicos de la zona o sector (sociales, sanitarios, educativos y de empleo) en el apoyo especializado a alumnos y centros. La muestra final, compuesta por 9732 sujetos, fue seleccionada a partir de un muestreo aleatorio proporcional al tamaño de las sub-poblaciones de cada Comunidad. Los resultados muestran cómo, en términos generales, existe colaboración entre el centro y los servicios públicos de la zona, aunque no tan frecuentemente como sería deseable. Los profesionales encuestados consideran que la colaboración con los servicios sociales y educativos es bastante adecuada, pero la valoración no es tan positiva cuando se analizan los servicios de salud y los servicios de empleo. Finalmente, atendiendo a las diferentes profesionales considerados, son los tutores de ambos niveles educativos quienes manifiestan un mayor grado de adecuación de la colaboración entre el centro y los servicios públicos de la zona, exceptuando los servicios sociales.

Palabras clave: orientación educativa, cooperación interinstitucional, relación escuelacomunidad, educación primaria, educación secundaria. 


\begin{abstract}
The provision of guidance and educational support requires joint work and the collaboration of different professionals and institutions, especially when we face complex problems that require a high level of specialization and the combination of knowledge from different areas. The research has aimed to examine the proximity of the institutional system of guidance and support to school in nine Autonomous Communities, to the intersectorial approach of counselling. We present the results of a descriptive study using the survey method, which allows knowing the opinions of counsellors, tutors and principals of Primary and Secondary Compulsory Education about the collaboration with the local public services (social, health, education, and employment) in the specialized support to students and schools. The final sample consisted of 9732 subjects who were selected from a random sampling proportional to the size of the subpopulations of each Autonomous Community. Results indicate how, in general terms, there is collaboration among the school and the local public services, although not as frequently as it would be desirable. In the same way, the professionals that were interviewed believe that the collaboration with social and educational services is quite adequate, but the assessment is not as positive when health and employment services are analysed. Finally, taking into account the different professionals considered, tutors from both educational stages are the ones that show a higher degree of satisfaction with the collaboration between the school and the local public services, except in the case of social services.
\end{abstract}

Keywords: educational guidance, inter-institutional cooperation, school community cooperation, primary education, secondary education.

La educación, entendida como el conjunto de prácticas sociales mediante las cuales, y gracias a las cuales, los grupos humanos promueven el desarrollo y la socialización de todos sus miembros, se configura como una responsabilidad que compete a la sociedad en su conjunto (Coll, 2004) y que no se limita a la escolarización (Vélaz-deMedrano, 2009). La persona, como ser social, se construye a partir de los vínculos que establece con los diferentes contextos de desarrollo. Este hecho hace que los parámetros de la acción psicoeducativa se vean ampliados y que ésta incorpore, mediante la toma de decisiones y corresponsabilidad participada y vinculante, a los diferentes agentes e instituciones educativas y sociales (Serra, 2008).

Desde esta perspectiva, la orientación se entiende como una actividad de ayuda, de naturaleza fundamentalmente educativa, cuyo principal objetivo es contribuir al desarrollo integral del alumno, con el fin de capacitarlo para un aprendizaje autónomo y una participación activa, crítica y transformadora de la sociedad (García, Moreno \& Torrego, 1993). Para ello, la actividad orientadora se desarrolla en diferentes contextos, lo que implica una consideración ecológico-sistémica de la intervención educativa (Álvarez Rojo, 1994; Vélaz-de-Medrano, 1998, 2002) y la concurrencia armonizada de diferentes profesionales. En esta línea, Cano Escoriaza y García Nieto (2009a) remarcan el consenso existente en la literatura, en relación a la necesidad de que el alumnado, las familias, la dirección y los distintos recursos comunitarios se integren en los centros teniendo en cuenta las necesidades demandadas por los distintos subsistemas. 
En las complejas sociedades en las que se desarrolla el individuo resultaría paradójico pensar en una intervención orientadora o apoyo psicopedagógico aislados, que no contase con los recursos que puede brindar la sociedad desde sus distintas instituciones, y que no previese la colaboración de diferentes profesionales y servicios especializados. Consecuentemente, y ante esta situación, el denominado modelo ecológico de la orientación (Conyne \& Cooks, 2004) se presenta como un sistema de ayuda contextualizada, dependiente del sujeto al que se destina y de su interacción con el ambiente. Este modelo considera la enorme influencia que tienen los ambientes en el desarrollo del sujeto, con los cuales establece una serie de relaciones constantes y recíprocas. De este modo, como apunta Roselló (1998), no cabe aislar cada una de las intervenciones académicas, psicopedagógicas, sociales, sanitarias y de inserción sociolaboral de los distintos agentes comunitarios, sino que se debe tener en cuenta el carácter sistémico del contexto en el que se desarrolla el acto educativo, que está inscrito en un sistema más amplio sobre el que hay que actuar de forma global. Por esta razón, esta autora defiende que debemos superar la tradicional división de la intervención orientadora en diferentes niveles y apostar por un modelo de intervención integral e integrado, que asuma el enfoque de la intervención desde un modelo teóricometodológico de corte ecológico-sistémico; la orientación, por tanto, debe abrirse a nuevos entornos sociocomunitarios (Hervás Avilés, 2006).

Partiendo de este enfoque, el sistema educativo español ha incorporado desde los años 90 un conjunto de profesionales (orientadores, personal técnico de servicios a la comunidad, especialistas en audición y lenguaje o en pedagogía terapéutica, educadores sociales) que, bien en los centros o desde los equipos de zona, prestan apoyo para mejorar la igualdad de oportunidades en el disfrute de una educación de calidad para todo el alumnado. En cualquier caso, este hecho no garantiza per se el desarrollo de una educación con carácter inclusivo; es prerrequisito, si no se quiere caer en la balcanización de actuaciones individuales, que esta acumulación de tareas, funciones y especialistas, multipliquen su capacidad de actuación, estableciendo sistemas de coordinación profesional fluidos en la comunicación, concluyentes en lo ontológico y resolutivos en la acción (San Fabián Maroto, 2006). En definitiva, se trata de constituir un sistema de refuerzo y apoyo a la educación (no solo a la escolarización) que permita a los centros escolares y a los profesionales de la educación extraer el valor añadido que puede aportar una educación de calidad al desarrollo del potencial de cada niño o joven, sin que las circunstancias adversas de partida lo impidan.

Desde este enfoque, el papel de la orientación y la figura del orientador constituyen elementos fundamentales que requieren la reconceptualización de la orientación, del papel del orientador y del alcance de su práctica (Vélaz-de-Medrano, 2008; McMahon, Mason, Daluga-Guenther \& Ruiz, 2014). De acuerdo con The National Center for Transforming School Counseling (NCTSC) (2009), la profesión de orientador escolar debe centrarse en las relaciones e interacciones entre los alumnos y el entorno escolar con el fin de reducir los efectos de posibles barreras contextuales e institucionales que pueden limitar el desarrollo del estudiante. En su trabajo sobre la nueva visión del orientador escolar, la NCTSC (2009) reconoce como uno de los elementos clave del trabajo de este profesional, la colaboración con otros agentes de ayuda, tales como 
compañeros, profesores, directores, agencias comunitarias, empresas, etc., así como la coordinación de servicios escolares y de la comunidad en la atención a alumnado, familias y profesionales.

Para que esta colaboración y coordinación con otros agentes y servicios resulte efectiva, adoptar un enfoque intersectorial en la provisión de orientación y apoyo escolar se convierte en algo no solo deseable sino, sobre todo, necesario. Bryson, Crosby y Middeleton (2006) definen esta perspectiva intersectorial de la educación como la vinculación o la puesta en común de información, recursos, actividades, y capacidades de las organizaciones en dos o más sectores para lograr un resultado que no podría ser alcanzado por un sector separado. El enfoque intersectorial llevaría implícita una voluntad de cambio promovida por el deseo de conciliar y coordinar los recursos especializados dando una respuesta integral, sin limitar dicha respuesta a la acción escolar (Vélaz-de-Medrano, 2013a).Por su parte, organismos internacionales como el Consejo de la Unión Europea (2004), reconocen esta necesidad, apuntando la conveniencia de reforzar el papel, la calidad y la coordinación de los sistemas y servicios de información y orientación, así como el impulso de asociaciones a todos los niveles (nacional, regional, local y sectorial), reconociendo la responsabilidad compartida por parte de los diferentes agentes. En el marco de los principios establecidos en el preámbulo de la Ley Orgánica de Mejora de la Calidad Educativa (LOMCE, 2013), también se reconoce la importancia de la colaboración permanente y respetuosa de todos los actores de la educación; se enfatiza la necesidad de la autonomía de los centros como mecanismo para mantener la cohesión y la unidad del sistema, así como para abrir nuevas posibilidades de cooperación entre los centros y la creación de redes de apoyo y aprendizaje compartido. Para ello, entre las competencias del director, se establece colaborar con las familias, con las instituciones y con organismos que faciliten la relación del centro con el entorno, y fomentar un clima escolar que favorezca el estudio y el desarrollo de cuantas actuaciones propicien una formación integral. Además, se les encomienda fijar las directrices para la colaboración, con fines educativos y culturales, con las Administraciones locales y con otros centros, entidades y organismos (LOMCE, Artículo 132).

El enfoque intersectorial, contribuye a ofrecer una atención especializada y contextualizada, a partir de la mejora de la conexión y la coordinación de los distintos servicios (Vélaz-de-Medrano, 2009). Si se pretende posibilitar una intervención orientadora de más calidad, las escuelas deben ser concebidas como centros abiertos al contexto que permitan situar la acción orientadora en la comunidad en la que está inmersa. Solo de este modo podrán garantizarse la continuidad de las actuaciones (Longas \& Molla, 2007) y la coherencia del proceso educativo y orientador. Tal y como apunta Rodríguez Romero (1996), el propósito debe ser crear una identidad colectiva permitiendo la intersubjetividad, reforzando los vínculos que el individualismo y el aislamiento nos han hecho desdibujar. Esto supone la necesidad de cambiar de planteamiento; requiere pasar de considerar a la población como mera receptora, usuaria o destinataria, a convertirla en protagonista activa y en agente de transformación del propio proceso educativo. 
Este cambio es importante, pero conformar un verdadero sistema integral de apoyo a la educación es una tarea compleja que implica un alto grado de colaboración multiprofesional e interdisciplinar, y que precisa del trabajo conjunto de los orientadores y tutores con los diferentes servicios públicos, tales como los servicios sociales, de salud, educativos y de empleo. En este sentido, una de las principales dificultades de la colaboración intersectorial entre los distintos agentes implicados estriba en romper las rígidas barreras que suelen establecerse en torno a cada especialidad. Es por este motivo que debe entenderse que ello no supone una reducción de la capacidad de respuesta de cada profesional, sino una suma de esfuerzos y saberes en virtud de la consecución de un mismo objetivo (Bassedas, 2011). El establecimiento de una acción común, permite evitar un posible fenómeno en el que se difuminen responsabilidades, permitiendo un compromiso compartido estructurado en base a un objetivo común, sin olvidar por ello las diferencias entre los distintos perfiles personales y profesionales, manteniendo la capacidad de respuesta de cada uno de ellos y fortaleciendo, al mismo tiempo, la acción común.

Junto a la dificultad de romper barreras, Vélaz-de-Medrano (2009) identifica como una de las debilidades más importantes del actual sistema de apoyo a los centros escolares, el aislamiento en el que siguen viviendo estas instituciones, muchas veces cerradas y aisladas en sí mismas, así como las dificultades existentes para que los profesores, equipos directivos, familias y recursos del entorno puedan intercambiar conocimientos y experiencias. Precisamente, el enfoque intersectorial aboga por un nuevo modelo de organización de la educación fundamentada en el compromiso y la corresponsabilidad de los agentes educativos, sociales y sanitarios que conforman su comunidad de referencia. El papel de orientadores, directores y tutores destaca, dentro de este modelo, por su trabajo a lo largo de distintos niveles, así como por su atención a las múltiples demandas del centro y su contacto con el funcionamiento global del mismo. Tal y como apuntan McMahon, Mason, Daluga-Guenther y Ruiz (2014), el orientador suele moverse a niveles de trabajo micro (en contacto con los alumnos), meso (trabajando con grupos de alumnos y de tutores) y macro (trabajando con gestores, servicios, organizaciones, etc.). Por su parte, el tutor, debido a que se le supone una gran implicación en el desarrollo integral del alumno, así como a su profundo conocimiento del grupo-clase y de cada uno de sus miembros, se constituye como un actor destacado en el enfoque intersectorial. Finalmente, dentro de este contexto, los directores aparecen como los principales facilitadores educativos de estas comunidades de aprendizaje, constituyendo el enlace entre los recursos de la comunidad y el centro (Wilmore, 2002).

\section{Objetivos de investigación}

A pesar de la importancia manifiesta de adoptar un enfoque intersectorial en la provisión de orientación y apoyo escolar que permita dar una respuesta integral a las necesidades del alumnado, son pocas las evidencias empíricas que constatan la existencia de esta colaboración entre los diferentes sectores implicados en el ámbito de la orientación escolar. Conocer esta realidad, por tanto, se convierte en una tarea indispensable que posibilitará dibujar un mapa de las estructuras de orientación 
educativa disponibles a distintos niveles y, de esta forma, evitar la atomización de los recursos (Manzanares Moya, 2013).

Este trabajo de investigación busca contribuir al establecimiento de esta panorámica actualizada de las estructuras de orientación disponibles en el territorio nacional y, para ello, analiza la colaboración de los centros educativos de nueve Comunidades Autónomas con los distintos servicios comunitarios: sociales, de salud, educativos y de empleo. Concretamente, se ha buscado conocer la existencia de colaboración entre el centro y los servicios públicos de la zona, la adecuación de dicha colaboración, y la frecuencia con la que ambos agentes educativos coordinan sus actuaciones para atender los problemas del alumnado y de sus familias de manera integral. El estudio de dicha colaboración se ha llevado a cabo desde una doble perspectiva: el agente implicado y la etapa educativa (Primaria / Educación Secundaria Obligatoria).

\section{Método}

\section{Población y muestra}

El presente estudio se enmarca en el contexto del proyecto de investigación titulado "Los modelos institucionales de Orientación educativa en las Comunidades Autónomas (CCAA) españolas", financiado por el Ministerio de Ciencia e Innovación en el marco del Plan Nacional I+D+i 2008-2011 (Ref. EDU2008-06389/EDUC). En dicha investigación se llevó a cabo un estudio cuantitativo mediante encuesta dirigida a los directores, orientadores y tutores de Educación Primaria (E.P.) y Educación Secundaria Obligatoria (E.S.O)de nueve Comunidades Autónomas: Andalucía, Canarias, Cantabria, Castilla La Mancha, Cataluña, Galicia, Madrid, Navarra y País Vasco. El considerar la percepción de estas figuras profesionales y no de otros miembros de la comunidad educativa se debe, fundamentalmente, a que estos agentes son los responsables directos del sistema de orientación y apoyo escolar de su centro.

La selección de la muestra de este estudio se llevó a cabo a través de un muestreo aleatorio proporcional al tamaño de las sub-poblaciones de cada Comunidad. Las poblaciones de referencia las constituyeron los centros educativos (unidad primaria de muestreo) de E.P. $(\mathrm{N}=7232)$ y de E.S.O. $(\mathrm{N}=2500)$ de las nueve Comunidades Autónomas. En cada etapa, las poblaciones y las muestras fueron definidas por separado con el objetivo de disponer de datos representativos de cada una de ellas. Para la selección de los profesionales implicados, no se llevó a cabo un muestreo de segunda etapa, ya que las figuras del orientador y el director son únicas por centros. La selección de los tutores se realizó tomando en los centros de Educación Primaria a un tutor de cada ciclo, y en los centros de Educación Secundaria Obligatoria a dos tutores, un tutor de $2^{\circ}$ de E.S.O y otro de $4^{\circ}$ de E.S.O, más uno de Diversificación Curricular y otro de Programas de Cualificación Profesional Inicial (PCPI) ${ }^{1}$ cuando se ofertaban tales enseñanzas en el centro.

\footnotetext{
${ }^{1}$ Tras la promulgación de la LOMCE (2013), los Programas de Diversificación Curricular (PDC) se han sustituido por los Programas de Mejora del Aprendizaje, y los Programas de Cualificación Profesional Inicial (PCPI) por la Formación Profesional Básica. 
La Tabla 1 presenta el tamaño y la composición de la muestra final de las muestras obtenidas en este estudio, para cada etapa y Comunidad Autónoma.

\begin{tabular}{|c|c|c|c|c|c|c|}
\hline & \multicolumn{3}{|c|}{ E.P. } & \multicolumn{3}{|c|}{ E.S.O. } \\
\hline & Directores & Orientadores & Tutores & Directores & Orientadores & Tutores \\
\hline Andalucía & 103 & * & 307 & 111 & 109 & 365 \\
\hline Cantabria & 6 & 5 & 19 & 6 & 6 & 20 \\
\hline Cataluña & 84 & * & 246 & 65 & 59 & 170 \\
\hline $\begin{array}{l}\text { Castilla La } \\
\text { Mancha }\end{array}$ & 34 & 33 & 101 & 29 & 29 & 103 \\
\hline Canarias & 35 & * & 93 & 23 & 22 & 72 \\
\hline Galicia & 36 & 36 & 112 & 31 & 32 & 107 \\
\hline Madrid & 37 & $*$ & 108 & 39 & 38 & 119 \\
\hline Navarra & 9 & 9 & 24 & 5 & 5 & 14 \\
\hline País Vasco & 18 & 15 & 52 & 15 & 17 & 46 \\
\hline TOTAL & 362 & 98 & 1062 & 324 & 317 & 1016 \\
\hline
\end{tabular}

* No se cuenta con una estructura (unidad o departamento) interna de orientación en los centros de E.P.

Tabla 1. Muestra final por etapa y figura.

\section{Variables medidas e instrumentos aplicados}

La técnica de indagación empleada en este estudio fue la encuesta de tipo sociológico. Durante el curso 2013/14 se aplicaron diferentes cuestionarios, adaptados a las tres figuras (directores, tutores y orientadores) de las dos etapas (Educación Primaria y Educación Secundaria Obligatoria) de cada una de las nueve Comunidades Autónomas. Para la construcción de los cuestionarios se consideraron los resultados de un estudio cualitativo previo (Vélaz-de-Medrano, 2013b), en el que se estudiaron las políticas públicas de orientación educativa de seis comunidades autónomas. En una segunda fase, un panel compuesto por expertos en orientación educativa, llevó a cabo la validación de contenido de los instrumentos elaborados. En tercer lugar, profesionales de la orientación de las nueve Comunidades Autónomas, revisaron los cuestionarios, incorporando las peculiaridades estructurales de la orientación y, por tanto, terminológicas, de cada una de ellas.

En el caso concreto del análisis de la dimensión intersectorial y comunitaria de la provisión de orientación y apoyo a la escuela, se ha tenido en cuenta, exclusivamente, la respuesta otorgada por los directores, orientadores y tutores de la muestra a las siguientes cuestiones:

- ¿Existe colaboración entre el centro y los servicios públicos de la zona (servicios sociales, de salud, educativos y de empleo) para atender a los 
problemas específicos del alumnado y sus familias? Pregunta de respuesta cerrada con dos categorías de respuesta (1: No; 2 : Sí).

- Valore la colaboración entre el centro y los distintos servicios públicos de la zona: 1) Servicios sociales, 2) Servicios de salud, 3) Servicios educativos y 4) Servicios de empleo. Pregunta de respuesta cerrada tipo Likert de 6 puntos (1: Nada adecuada; 6: Completamente adecuada).

- ¿Con qué frecuencia esos servicios públicos coordinan sus actuaciones entre sí y con el centro para atender los problemas del alumnado y sus familias de manera integral? Pregunta de respuesta cerrada tipo Likert de 6 puntos (1: Nunca; 6: Siempre).

Es importante señalar que estas preguntas son comunes a la totalidad de instrumentos elaborados, a excepción de la cuestión relativa a la frecuencia con la que los servicios públicos coordinan sus actuaciones entre sí y con el centro, que solo se incluyó en los cuestionarios dirigidos a los directores de E.P. y a los directores y orientadores de E.S.O.

\section{Análisis de datos}

El análisis de los resultados se ha llevado a cabo desde una doble aproximación, descriptiva e inferencial. En este sentido, en primer lugar, se estudia la distribución de la respuesta otorgada a cada una de las cuestiones planteadas y, a continuación se procede a analizar posibles diferencias en la percepción que los diferentes profesionales encuestados tienen respecto a la colaboración entre el centro educativo y los servicios públicos de la zona en función de la figura profesional y de la etapa educativa. En esta segunda fase, se ha adoptado un enfoque no paramétrico debido a la naturaleza cualitativa de las variables objeto de estudio (variables nominales $u$ ordinales), calculando el estadístico más adecuado en función de las características de las variables implicadas (Chi Cuadrado, U de Mann-Whitney y H de Kruskal-Wallis).

\section{Resultados}

\section{Colaboración entre el centro y los servicios públicos de la zona}

En relación con la existencia de colaboración entre el centro y los servicios públicos de la zona para atender a los problemas específicos del alumnado y sus familias, el Gráfico 1 muestra cómo el 96,1\% de los profesionales encuestados de E.P. y el 97,1\% de los directores, orientadores y tutores de E.S.O. coinciden en afirmar que, en general, sí existe colaboración entre el centro y los servicios públicos. Esta misma tendencia se pone de manifiesto en la respuesta que han otorgado los diferentes profesionales de las dos etapas educativas consideradas. 


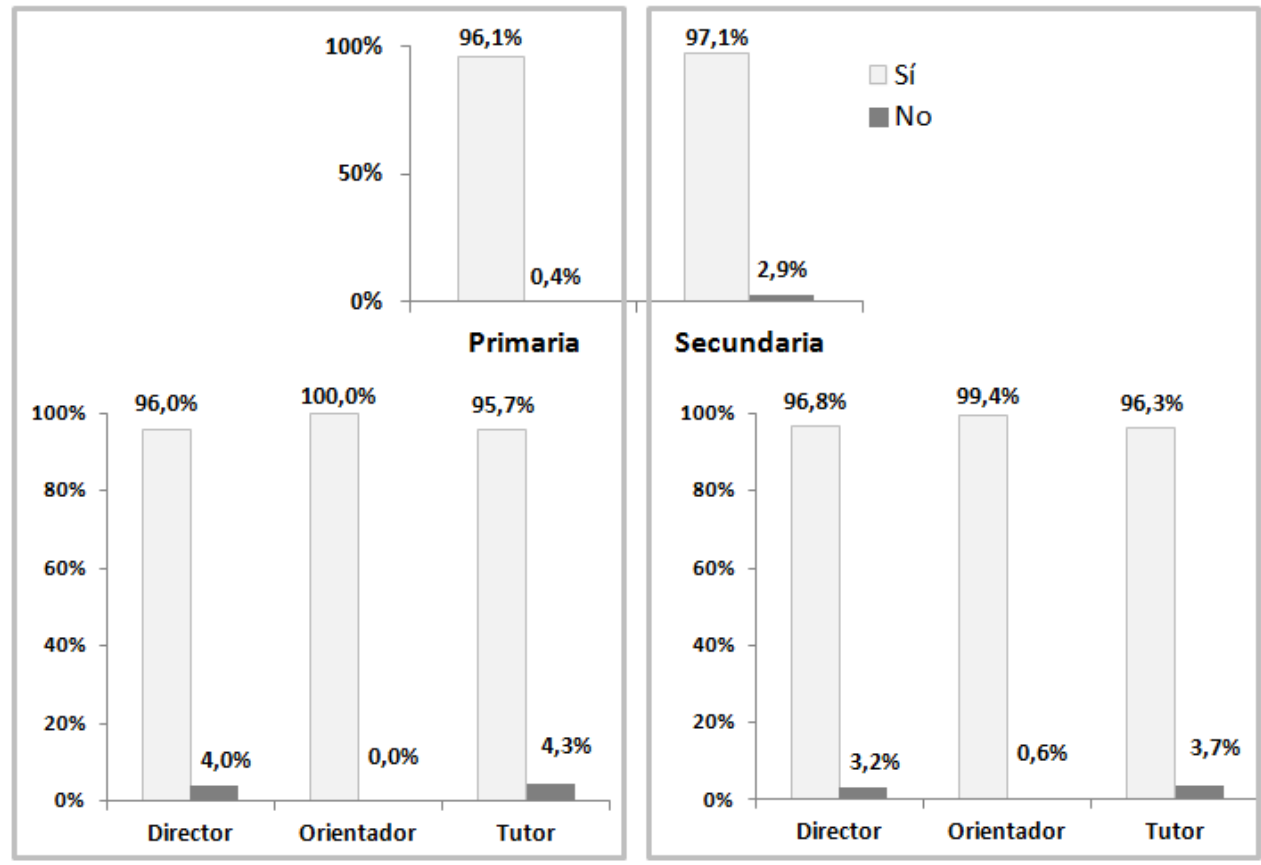

Grafico 1. Existencia de colaboración entre los centros y los servicios públicos de la zona

A pesar del amplio consenso en la valoración positiva de dicha colaboración, se ha llevado a cabo a través de la prueba Chi Cuadrado un análisis de las posibles diferencias en la respuesta proporcionada por los sujetos que forman parte de la muestra en función de la etapa educativa y de la figura profesional. Los resultados (Tabla 2), revelan la existencia de diferencias estadísticamente significativas tan solo en el caso de los profesionales de Educación Secundaria, siendo los orientadores los que en mayor medida indican que existe colaboración entre el centro y los servicios públicos de la zona.

\begin{tabular}{|c|c|c|c|c|c|c|c|c|c|c|}
\hline & & \multicolumn{3}{|c|}{ E.P. } & \multicolumn{3}{|c|}{ E.S.O. } & \multicolumn{3}{|c|}{ Diferencias entre etapas } \\
\hline & & D & $\mathbf{O}$ & $\mathbf{T}$ & D & O & $\mathbf{T}$ & D & O & $\mathbf{T}$ \\
\hline \multirow{2}{*}{ SÍ } & $\mathbf{N}$ & 340 & 91 & 775 & 306 & 307 & 731 & \multirow{5}{*}{$\begin{array}{l}\chi^{2}: 0,302 \\
\text { p. } 0,583\end{array}$} & \multirow{5}{*}{$\begin{array}{l}\chi^{2}: 0,592 \\
\text { p. } 0,442\end{array}$} & \multirow{5}{*}{$\begin{array}{l}\chi^{2}: 0,406 \\
\text { p. } 0,524\end{array}$} \\
\hline & $\%$ & $96,0 \%$ & $100 \%$ & $95,7 \%$ & $96,8 \%$ & $99,4 \%$ & $96,3 \%$ & & & \\
\hline \multirow{2}{*}{ NO } & $\mathbf{N}$ & 14 & 0 & 35 & 10 & 2 & 28 & & & \\
\hline & $\%$ & $4,0 \%$ & $0 \%$ & $4,3 \%$ & $3,2 \%$ & $0,6 \%$ & $3,7 \%$ & & & \\
\hline \multicolumn{2}{|c|}{$\begin{array}{l}\text { Diferencias } \\
\text { entre figuras }\end{array}$} & \multicolumn{3}{|c|}{$\begin{array}{c}\chi^{2}: 4,074 \\
\text { p. } 0,13\end{array}$} & \multicolumn{3}{|c|}{$\begin{array}{l}\chi^{2}: 7,349 \\
\text { p. } 0,025\end{array}$} & & & \\
\hline
\end{tabular}

Nota: $\mathrm{D}=$ Director; $\mathrm{O}=$ Orientador; $\mathrm{T}=$ Tutor.

Tabla 2. Existencia de colaboración entre el centro y los servicios públicos en función de la etapa y la figura profesional. 


\section{Adecuación de la colaboración entre el centro y los distintos servicios públicos}

Considerando los sujetos de la muestra que han afirmado que existe colaboración entre el centro y los servicios públicos de la zona, se ha estudiado su percepción respecto al grado de adecuación de dicha colaboración entre el centro y los servicios sociales, los servicios de salud, los servicios educativos y los servicios de empleo. En la Tabla 3, en primer lugar, se destaca una alta valoración a la colaboración existente entre los centros educativos y los servicios sociales por parte de los diferentes profesionales de las dos etapas educativas. En este sentido, más del $60 \%$ de los profesionales encuestados consideran que dicha colaboración es "bastante" o "completamente adecuada". En Educación Secundaria Obligatoria, este porcentaje toma valores próximos al $70 \%$.

Con una distribución de respuesta similar a la anteriormente descrita, aparece la valoración del nivel de adecuación de la colaboración entre el centro y otros servicios educativos de la zona. En todos los casos, el porcentaje de sujetos que apuntan que dicha colaboración es "bastante" o "completamente" adecuada toma valores superiores al $50 \%$.

En relación con los servicios de salud, aunque el número de profesionales que opinan que la colaboración es "bastante" o "completamente adecuada" es elevado, existe un amplio porcentaje de sujetos que indican que se colabora "poco" o "algo", es decir, que sitúan en el nivel central de la escala la cooperación entre el centro y los servicios de salud de la zona. Atendiendo a la valoración otorgada por los orientadores de Educación Secundaria Obligatoria, las respuestas que se sitúan en este nivel, alcanzan valores superiores al $50 \%$.

Finalmente, la tendencia se invierte en el caso de los servicios de empleo, especialmente en la etapa de E.P. En dicho nivel educativo, los profesionales que forman parte de la muestra consideran que la colaboración con estos servicios no es adecuada. En este sentido, destaca la respuesta del 66,9\% de los Directores y del 76,7\% de los Orientadores que la valoran como "nada" o "casi nada" adecuada. En E.S.O., aunque es ligeramente superior la percepción del nivel de adecuación de la colaboración entre el centro y los servicios de empleo, la mayor parte de los sujetos coinciden en clasificarla como "poco" o "algo" adecuada. 


\begin{tabular}{|c|c|c|c|c|c|c|c|}
\hline & & \multicolumn{3}{|c|}{ E.P. } & \multicolumn{3}{|c|}{ E.S.O. } \\
\hline & & Director & Orientador & Tutor & Director & Orientador & Tutor \\
\hline \multirow{3}{*}{$\begin{array}{l}\text { SERVICIOS } \\
\text { SOCIALES }\end{array}$} & Nada / Casi nada & $3,6 \%$ & $6,7 \%$ & $2,0 \%$ & $2,3 \%$ & $1,7 \%$ & $2,0 \%$ \\
\hline & Poco / Algo & $31,2 \%$ & $30,0 \%$ & $34,6 \%$ & $24,7 \%$ & $31,0 \%$ & $25,3 \%$ \\
\hline & $\begin{array}{l}\text { Bastante / } \\
\text { Completamente }\end{array}$ & $65,3 \%$ & $63,3 \%$ & $63,5 \%$ & $73,0 \%$ & $67,3 \%$ & $72,7 \%$ \\
\hline \multirow{3}{*}{$\begin{array}{l}\text { SERVICIOS } \\
\text { DE SALUD }\end{array}$} & Nada / Casi nada & $14,5 \%$ & $14,6 \%$ & $8,9 \%$ & $11,4 \%$ & $12,8 \%$ & $7,0 \%$ \\
\hline & Poco / Algo & $42,2 \%$ & $49,4 \%$ & $42,9 \%$ & $45,5 \%$ & $52,0 \%$ & $39,9 \%$ \\
\hline & $\begin{array}{l}\text { Bastante / } \\
\text { Completamente }\end{array}$ & $43,4 \%$ & $36,0 \%$ & $48,2 \%$ & $43,1 \%$ & $35,2 \%$ & $53,1 \%$ \\
\hline \multirow{3}{*}{$\begin{array}{l}\text { SERVICIOS } \\
\text { EDUCATIVOS }\end{array}$} & Nada / Casi nada & $5,8 \%$ & $6,4 \%$ & $2,3 \%$ & $4,1 \%$ & $5,8 \%$ & $5,1 \%$ \\
\hline & Poco / Algo & $34,1 \%$ & $42,3 \%$ & $33,7 \%$ & $32,8 \%$ & $41,6 \%$ & $31,7 \%$ \\
\hline & $\begin{array}{l}\text { Bastante / } \\
\text { Completamente }\end{array}$ & $60,1 \%$ & $51,3 \%$ & $64,0 \%$ & $63,2 \%$ & $52,6 \%$ & $63,1 \%$ \\
\hline \multirow{3}{*}{$\begin{array}{l}\text { SERVICIOS } \\
\text { DE EMPLEO }\end{array}$} & Nada / Casi nada & $66,9 \%$ & $76,7 \%$ & $46,4 \%$ & $33,1 \%$ & $30,4 \%$ & $18,7 \%$ \\
\hline & Poco / Algo & $25,3 \%$ & $16,4 \%$ & $40,6 \%$ & $48,9 \%$ & $52,2 \%$ & $44,6 \%$ \\
\hline & $\begin{array}{l}\text { Bastante / } \\
\text { Completamente }\end{array}$ & $7,8 \%$ & $6,8 \%$ & $13,0 \%$ & $18,0 \%$ & $17,4 \%$ & $36,7 \%$ \\
\hline
\end{tabular}

Tabla 3. Adecuación de colaboración con los distintos servicios públicos.

El análisis de las diferencias en la valoración otorgada por los profesionales en función de la etapa educativa, se ha llevado a cabo a través de la prueba U de MannWhitney. Los resultados (Tabla 4) muestran cómo, en relación con los servicios sociales, los directores de E.P. consideran ligeramente menos adecuada la colaboración existente, que los directores de E.S.O., siendo las diferencias estadísticamente significativas. Esta misma inclinación se observa en el caso de los tutores.

Atendiendo a los servicios de salud, si se analiza la respuesta otorgada por los diferentes profesionales encuestados en función de la etapa educativa, se muestra cómo en el caso de los directores y los orientadores, la valoración es ligeramente superior en la etapa de Educación Secundaria, aunque dichas diferencias no son estadísticamente significativas. Por su parte, la valoración de los tutores de Secundaria, es significativamente superior que la de sus compañeros de Primaria. 


\begin{tabular}{|c|c|c|c|c|c|c|c|c|c|c|}
\hline & & \multicolumn{3}{|c|}{ Director } & \multicolumn{3}{|c|}{ Orientador } & \multicolumn{3}{|c|}{ Tutor } \\
\hline & & E.P. & E.S.O. & Diferencias & E.P. & E.S.O. & Diferencias & E.P. & E.S.O. & Diferencias \\
\hline \multirow{3}{*}{$\begin{array}{l}\text { SERVICIOS } \\
\text { SOCIALES }\end{array}$} & $1^{\mathrm{er}}$ & 4 & 4 & \multirow{2}{*}{$\begin{array}{c}\mathrm{U}: 46652,5 \\
\text { p.: } 0,031\end{array}$} & 4 & 4 & \multirow{2}{*}{$\begin{array}{c}\mathrm{U}: 12804,0 \\
\text { p.: } 0,337\end{array}$} & 4 & 4 & \multirow{2}{*}{$\begin{array}{c}\mathrm{U}: 219264,0 \\
\text { p.: } 0,00\end{array}$} \\
\hline & $2^{\circ}$ & 5 & 5 & & 5 & 5 & & 5 & 5 & \\
\hline & $3^{\text {er }}$ & 5 & 5 & $\mathrm{EP}<\mathrm{ES}$ & 5 & 5 & $\mathrm{EP}=\mathrm{ES}$ & 5 & 5 & $\mathrm{EP}<\mathrm{ES}$ \\
\hline \multirow{3}{*}{$\begin{array}{l}\text { SERVICIOS } \\
\text { DE SALUD }\end{array}$} & $1^{\mathrm{er}}$ & 3 & 4 & \multirow{2}{*}{$\begin{array}{c}\mathrm{U}: 45867,5 \\
\text { p.: } 0,208\end{array}$} & 3 & 3 & \multirow{2}{*}{$\begin{array}{c}\mathrm{U}: 13469,5 \\
\text { p.: } 0,949\end{array}$} & 4 & 4 & \multirow{2}{*}{$\begin{array}{c}\mathrm{U}: 194653,0 \\
\text { p.: } 0,015\end{array}$} \\
\hline & $2^{\circ}$ & 4 & 4 & & 4 & 4 & & 4 & 5 & \\
\hline & $3^{\text {er }}$ & 5 & 5 & $\mathrm{EP}=\mathrm{ES}$ & 5 & 5 & $\mathrm{EP}=\mathrm{ES}$ & 5 & 5 & $\mathrm{EP}<\mathrm{ES}$ \\
\hline \multirow{3}{*}{$\begin{array}{l}\text { SERVICIOS } \\
\text { EDUCATIVOS }\end{array}$} & $1^{\mathrm{er}}$ & 4 & 4 & \multirow{2}{*}{$\begin{array}{c}\mathrm{U}: 43841,0 \\
\text { p.: } 0,270\end{array}$} & 4 & 4 & \multirow{2}{*}{$\begin{array}{c}\mathrm{U}: 11074,0 \\
\text { p.: } 0,727\end{array}$} & 4 & 4 & \multirow{2}{*}{$\begin{array}{c}\mathrm{U}: 108874,5 \\
\text { p.: } 0,536\end{array}$} \\
\hline & $2^{o}$ & 5 & 5 & & 5 & 5 & & 5 & 5 & \\
\hline & $3^{\text {er }}$ & 5 & 5 & $\mathrm{EP}=\mathrm{ES}$ & 5 & 5 & $\mathrm{EP}=\mathrm{ES}$ & 5 & 5 & $\mathrm{EP}=\mathrm{ES}$ \\
\hline \multirow{3}{*}{$\begin{array}{l}\text { SERVICIOS } \\
\text { DE EMPLEO }\end{array}$} & $1^{\mathrm{er}}$ & 2 & 3 & \multirow{2}{*}{$\begin{array}{c}\mathrm{U}: 23959,5 \\
\text { p.: } 0,000\end{array}$} & 2 & 3 & \multirow{2}{*}{$\begin{array}{l}\text { U: } 5282,0 \\
\text { p.: } 0,000\end{array}$} & 3 & 4 & \multirow{2}{*}{$\begin{array}{c}\text { U: } 88329,5 \\
\text { p.: } 0,000\end{array}$} \\
\hline & $2^{\circ}$ & 1 & 2 & & 1 & 2 & & 2 & 3 & \\
\hline & $3^{\text {er }}$ & 3 & 4 & $\mathrm{EP}<\mathrm{ES}$ & 2 & 4 & $\mathrm{EP}<\mathrm{ES}$ & 4 & 5 & $\mathrm{EP}<\mathrm{ES}$ \\
\hline
\end{tabular}

Tabla 4. Adecuación de colaboración con los distintos servicios públicos en función de la etapa educativa.

Con respecto a la colaboración con los servicios educativos, las diferencias en la percepción que los directores, orientadores y tutores tienen respecto a la adecuación de la colaboración entre los centros y dichos servicios públicos de la zona, no difieren en función de la etapa educativa considerada.

Finalmente, al considerar la valoración del grado de adecuación de la colaboración con los servicios de empleo se observa cómo, la valoración de los directores de E.S.O. es superior a la que expresan sus compañeros de E.P., resultando esta diferencia estadísticamente significativas. En la respuesta de los orientadores y tutores, también la valoración es significativamente superior en la etapa de E.S.O.

Con el objetivo de analizar si la valoración de la adecuación de la colaboración difiere en función de la figura profesional, se ha utilizado la prueba $\mathrm{H}$ de KruskalWallis. Los resultados presentados en la Tabla 5 muestran cómo las diferencias en el grado de cooperación entre el centro y los servicios sociales percibido por directores, orientadores y tutores no resultan significativas, en ninguna de las dos etapas educativas consideradas. 


\begin{tabular}{|c|c|c|c|c|c|c|c|c|c|c|c|c|c|c|}
\hline & & \multicolumn{3}{|c|}{ Director } & \multicolumn{3}{|c|}{ Orientador } & \multicolumn{3}{|c|}{ Tutor } & \multirow[b]{2}{*}{$\chi^{2}$} & \multicolumn{3}{|c|}{ Comparaciones múltiples } \\
\hline & & $1^{\mathrm{er}}$ & $2^{\circ}$ & $3^{\text {er }}$ & $1^{\mathrm{er}}$ & $2^{\circ}$ & $3^{\text {er }}$ & $1^{\mathrm{er}}$ & $2^{\circ}$ & $3^{\text {er }}$ & & D & $\mathbf{O}$ & $\mathbf{T}$ \\
\hline \multirow{4}{*}{ 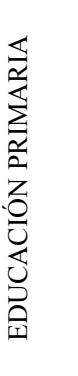 } & $\begin{array}{l}\text { Servicios } \\
\text { sociales }\end{array}$ & 4 & 5 & 5 & 4 & 5 & 5 & 4 & 5 & 5 & $\begin{array}{c}0,736 \\
\text { p. } 0,692\end{array}$ & & & \\
\hline & $\begin{array}{l}\text { Servicios de } \\
\text { salud }\end{array}$ & 3 & 4 & 5 & 3 & 4 & 5 & 4 & 4 & 5 & $\begin{array}{c}9,790 \\
\text { p. } 0,007\end{array}$ & & & $\mathrm{D}^{*} / \mathrm{O}^{*}$ \\
\hline & $\begin{array}{l}\text { Servicios } \\
\text { educativos }\end{array}$ & 4 & 5 & 5 & 4 & 5 & 5 & 4 & 5 & 5 & $\begin{array}{c}6,800 \\
\text { p. } 0,033\end{array}$ & & & $\mathrm{O}^{*}$ \\
\hline & $\begin{array}{l}\text { Servicios de } \\
\text { empleo }\end{array}$ & 1 & 2 & 3 & 1 & 2 & 2 & 2 & 3 & 4 & $\begin{array}{l}41,406 \\
\text { p. } 0,000\end{array}$ & & & $\mathrm{D}^{* *} / \mathrm{O}^{* *}$ \\
\hline \multirow{4}{*}{ 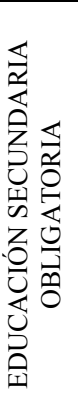 } & $\begin{array}{l}\text { Servicios } \\
\text { sociales }\end{array}$ & 4 & 5 & 5 & 4 & 5 & 5 & 4 & 5 & 5 & $\begin{array}{c}2,658 \\
\text { p. } 0,265\end{array}$ & & & \\
\hline & $\begin{array}{l}\text { Servicios de } \\
\text { salud }\end{array}$ & 4 & 4 & 5 & 3 & 4 & 5 & 4 & 5 & 5 & $\begin{array}{l}32,325 \\
\text { p. } 0,000\end{array}$ & $\mathrm{O}^{*}$ & & $\mathrm{D}^{* *} / \mathrm{O}^{* *}$ \\
\hline & $\begin{array}{l}\text { Servicios } \\
\text { educativos }\end{array}$ & 4 & 5 & 5 & 4 & 5 & 5 & 4 & 5 & 5 & $\begin{array}{c}7,233 \\
\text { p. } 0,027\end{array}$ & $\mathrm{O}^{*}$ & & $\mathrm{O}^{*}$ \\
\hline & $\begin{array}{l}\text { Servicios de } \\
\text { empleo }\end{array}$ & 2 & 3 & 4 & 2 & 3 & 4 & 3 & 4 & 5 & $\begin{array}{l}79,828 \\
\text { p. } 0,000\end{array}$ & & & $\mathrm{D}^{* *} / \mathrm{O}^{* *}$ \\
\hline
\end{tabular}

Nota $^{1}: \mathrm{D}=$ Director, $\mathrm{O}=$ Orientador $\mathrm{y} \mathrm{T}=$ Tutor.

Nota $^{2}$ : Las comparaciones múltiples entre los diferentes subgrupos se han llevado a cabo a través de la prueba U de Mann-Whitney. En aquellos casos en los que las diferencias resultan estadísticamente significativas, debajo del subgrupo que está siendo comparado se indica el sub-grupo (o los sub-grupos) en el cuál la valoración media es significativamente inferior.

Nota ${ }^{3}:{ }^{*}$ Nivel del confianza del 95\%; ${ }^{* *}$ Nivel de confianza del 99\%

Tabla 5. Adecuación de colaboración con los distintos servicios públicos en función de la figura profesional.

En relación con la adecuación de la colaboración con los servicios de salud, el análisis de las diferencias muestra cómo, en ambas etapas, la valoración de los tutores es significativamente más positiva que la de los directores y orientadores. Del mismo modo, en Educación Secundaria Obligatoria, los directores tienen una percepción más positiva que los orientadores con respecto a la adecuación de la colaboración.

En tercer lugar, y atendiendo a la colaboración con servicios educativos de la zona, se observa que, en ambas etapas educativas, existen diferencias estadísticamente significativas en la valoración otorgada en función del agente encuestado. En E.P., esas diferencias se reducen a una mayor valoración del grado de adecuación de la colaboración por parte de los tutores, respecto a la respuesta ofrecida por los orientadores. En la etapa de E.S.O., los orientadores consideran que la colaboración con los servicios educativos, no es tan adecuada como indican directores y tutores. 
Por último, la percepción del nivel de adecuación de la colaboración entre el centro y los servicios de empleo de la zona es significativamente superior en el caso de los tutores, tanto en E.P. como en E.S.O.

Frecuencia en la coordinación de los servicios públicos entre sí y con el centro para atender los problemas del alumnado y sus familias

Ante la pregunta: ¿con qué frecuencia esos servicios públicos coordinan sus actuaciones entre sí y con el centro escolar para atender los problemas del alumnado y sus familias de manera integral?, en la Tabla 6 puede verse cómo más del $65 \%$ de los directores de ambas etapas educativas, consideran que dicha coordinación se lleva a cabo "a veces" o "frecuentemente". En el caso de los orientadores de E.S.O dicho porcentaje toma un valor del $72,8 \%$.

De otro lado, los profesionales que consideran que "nunca" o "casi nunca" los servicios públicos coordinan sus actuaciones entre sí y con el centro suponen, aproximadamente, un $12 \%$ de los sujetos de la muestra. En el polo opuesto, se situarían el 20,5\% de los directores de E P., el 21,5\% de los directores de E.S.O. y el 15,4\% de los orientadores de Educación Secundaria, que consideran que "casi siempre" o "siempre" se coordinan los diferentes servicios públicos entre sí y con el centro.

Centrando el análisis en las posibles variaciones entre etapas, las diferencias observadas en la valoración a esta cuestión por parte de los directores de E.P. en relación a la de sus colegas de E.S.O., no resultan significativas estadísticamente (U: 49594,0; p. 0,807). .

Por otra parte, dentro de la etapa de Educación Secundaria Obligatoria, la percepción de los orientadores no difiere de la ofrecida por los directores de esa etapa (U: 42403,5; p. 0,199).

\begin{tabular}{|c|c|c|c|c|}
\hline & & $\begin{array}{c}\text { Nunca / Casi } \\
\text { nunca }\end{array}$ & $\begin{array}{c}\text { A veces / } \\
\text { Frecuentemente }\end{array}$ & $\begin{array}{c}\text { Casi siempre } \\
\text { Siempre }\end{array}$ \\
\hline $\begin{array}{l}\text { EDUCACIÓN } \\
\text { PRIMARIA }\end{array}$ & Director & $13,0 \%$ & $66,6 \%$ & $20,5 \%$ \\
\hline $\begin{array}{l}\text { EDUCACIÓN } \\
\text { SECUNDARIA }\end{array}$ & Director & $12,9 \%$ & $65,6 \%$ & $21,5 \%$ \\
\hline OBLIGATORIA & Orientador & $11,7 \%$ & $72,8 \%$ & $15,4 \%$ \\
\hline
\end{tabular}

Tabla 6. Frecuencia en la coordinación de los servicios públicos entre sí y con el centro.

\section{Conclusiones}

La importancia de la dimensión integral de la acción orientadora a través de la colaboración de los profesionales y servicios de zona o sector es defendida y argumentada a nivel teórico por la literatura especializada desde hace décadas pero, en el caso de España, no disponemos de suficientes evidencias empíricas que nos proporcionen una panorámica actualizada de esta realidad. Este estudio pretende, desde 
una aproximación descriptiva y extensiva (con amplia muestra), contribuir a reducir dicha incertidumbre, a través del conocimiento de la opinión de los profesionales implicados.

Los principales resultados, ponen de manifiesto el acuerdo, prácticamente generalizado de orientadores, directores y tutores, en afirmar que los centros educativos colaboran con los servicios públicos de la zona. Presumiblemente por la estrecha vinculación del problema estudiado con sus funciones, los orientadores, fundamentalmente en la etapa de Educación Secundaria Obligatoria, son los profesionales que valoran de forma más positiva dicha colaboración. En cierto modo, este hecho parece respaldar el avance hacia la integración de los diferentes recursos comunitarios en un "sistema" de apoyo a la educación en el que exista una complementariedad de servicios, y una coordinación en la planificación de intervenciones y recursos.

Profundizando en la adecuación de esta colaboración, el estudio muestra una percepción bastante positiva de los diferentes profesionales, especialmente cuando se valora la cooperación del centro con los servicios sociales y los servicios educativos. No ocurre lo mismo, en el caso de otros servicios públicos como los servicios de salud $\mathrm{y}$, fundamentalmente, los servicios de empleo, donde se desvela la necesidad de un mayor trabajo conjunto. El hecho de que los profesionales encuestados otorguen valoraciones diferentes a la adecuación de la labor compartida con los diferentes servicios públicos, podría estar emplazando una debilidad en la provisión de orientación y apoyo escolar desde un enfoque intersectorial. Desde el enfoque intersectorial, la intervención orientadora no debería limitarse a la colaboración con una parte de los recursos comunitarios implicados, en este caso, con los servicios sociales y educativos, se trata de conseguir la armonización de las intervenciones de los distintos sectores y profesionales de la zona, modelo en el cual el profesional de la orientación juega un importante papel de catalizador. Una vez detectadas las necesidades, facilitar los cauces de comunicación y el desarrollo comunitario, formar y sensibilizar a los profesionales implicados y establecer objetivos y metas compartidas deberían considerarse líneas de actuación prioritarias.

Del mismo modo, otro resultado que sugiere la necesidad de una mayor colaboración entre el centro y los servicios públicos de la zona, lo encontramos en la valoración que, tanto los directores de Educación Primaria y Secundaria Obligatoria como los orientadores de esta última etapa educativa, hacen de la frecuencia con la que los servicios públicos se coordinan entre sí y con el centro; son escasos los profesionales encuestados que reconocen que dicha colaboración se lleva a cabo constantemente (es decir, casi siempre o siempre). Reconocer el enfoque intersectorial de la orientación supone una ampliación del horizonte orientador, en el que los esfuerzos de todos los agentes sociales se suman para alcanzar un objetivo común. En este sentido, el trabajo en red debe configurarse como la base de las intervenciones orientadoras y de apoyo escolar, destinadas a facilitar los intercambios y la cooperación multiprofesional entre los diferentes sectores (educativos, sanitarios, sociales, etc.). 
Como aboga Coll (2000), adoptar esta visión intersectorial comporta la exigencia de redefinir las funciones, competencias y responsabilidades de los diferentes escenarios y agentes educativos, así como la de potenciar el establecimiento de un compromiso entre todos ellos en torno a esta redefinición. Consecuentemente, las tareas y funciones de cada uno de los profesionales implicados en la intervención orientadora, en relación con la coordinación con los servicios públicos de la zona, deben estar claramente delimitadas, siendo complementarias y sin solapamientos. Asimismo, deben ser asumidas corresponsablemente por todos, fomentando la coordinación eficaz y un trabajo conjunto y compartido mediante planes de actuación globales, en el que cada agente juegue un papel fundamental, para conseguir mejorar la respuesta educativa que se ofrece a cada alumno, sin olvidar la importancia de la reflexión de todos y cada uno de los implicados y el uso de la evaluación como elemento de ayuda para la gestión adecuada de los recursos y la mejora de las intervenciones sociales y educativas planteadas (Nuñez, Crespo, Úcar y Berñe, 2014).

Combatir la fragmentación de actividades entre los distintos profesionales implicados en la educación y la orientación, es una actuación imprescindible para lograr una gestión más eficaz de los recursos educativos, permitiendo ofrecer entre todos una orientación adaptada a las necesidades específicas de cada estudiante y de cada familia, aportando una visión global en la que se valoren las singulares aportaciones de cada perfil profesional en busca de un objetivo común. Esta perspectiva sistémica y de trabajo colaborativo ha sido defendida por autores como Cano Escoriaza y Garcia Nieto (2009b) o Hammer, Ripper y Schenk (2014), entre otros. Estos últimos autores proponen un interesante ejemplo de cooperación del centro educativo con el mundo laboral o productivo y con otros organismos, combatiendo la fragmentación a través de la creación de objetivos comunes por medio de un plan general de orientación profesional integral.

Es preciso señalar que, el enfoque intersectorial de la orientación no pretende desdibujar perfiles profesionales, muy al contrario, su objetivo es el fomento de la cooperación con el fin de abordar de forma integral la compleja realidad educativa, permitiendo el fortalecimiento de la estructura de orientación y apoyo al alumnado y sus familias. Por tanto, se trata de una intervención contextualizada y especializada, en la que cada profesional contribuye a alcanzar un objetivo común, el desarrollo integral del individuo y la mejora de la calidad de la atención que se le brinda desde distintas instituciones.

El estudio presentado aporta un conocimiento de la situación del problema en nueve CCAA que consideramos contribuirá a avanzar en la investigación de un tema tan relevante para el diseño e implantación de políticas educativas (y orientadoras) desde un enfoque sistémico y territorial en el que la mirada de los problemas y soluciones para la educación no se limitan al ámbito de la escolarización.

En términos generales, los resultados de este estudio -nada desdeñables desde la perspectiva de la diversidad de profesionales y la amplitud de la muestra participanteparecen replicar las intuiciones, experiencias o estudios que han llevado a numerosos autores (San Fabián, 2006; Santana, 2006; Vélaz-de-Medrano, 2009, 2013a, 2013b; Bassedas, 2011; Manzanares Moya, 2013; Fernández-Larragueta et al, 2014, entre 
otros) a ser menos optimistas con respecto a la colaboración entre profesionales escolares y servicios comunitarios. Como es de rigor, esto nos llevará a profundizar más en el problema, triangulando metodologías de indagación directas y proyectivas, extensivas e intensivas. Así, entre las limitaciones del estudio, que al mismo tiempo pueden configurarse como futuras líneas de investigación, se encontraría la ausencia de un análisis subjetivo-intersubjetivo, en un ámbito como el del enfoque intersectorial en el que los significados sociales propios y compartidos presentan una importancia crucial. En este sentido, la profundización en los fenómenos más destacados utilizando una aproximación cualitativa, que permitiría una triangulación en relación a los datos obtenidos en el análisis cuantitativo, se constituye como una interesante línea de investigación. Por otro lado, profundizar abordando la perspectiva de las necesidades específicas de la familia o el profesorado, así como la incorporación de la valoración del alumnado, la familia y la administración, podrían ser aspectos considerados en futuros trabajos.

\section{Referencias bibliográficas}

ÁLVAREZ ROJO, V. (1994). La orientación educativa y acción orientadora. Relaciones entre la teoría y la práctica. Sevilla: EOS.

BASSEDAS, E. (2011). Orientación y trabajo intersectorial, en E. Martín e I. Solé (Coords.), Orientación educativa. Modelos y estrategias de intervención, 169-186. Barcelona: Grao.

BRYSON, Y., CROSBY, B. \& MIDDELETON, M. (2006). The Design and Implementation of Cross-Sector Collaborations: Propositions from the Literature. Public Administration Review, 66 (1), 44-55.

CANO ESCORIAZA, J., \& GARCÍA NIETO, N. (2009a). Aproximación a la percepción de los orientadores escolares sobre la colaboración que reciben de la comunidad educativa: retos y propuestas. Revista Complutense de Educación, Vol.21, 1, 149-169.

CANO ESCORIAZA, J., \& GARCÍA NIETO, N. (2009b). Percepción del orientador acerca de la importancia que atribuye la comunidad educativa a sus funciones. Contextos educativos, 12, 57-75.

COLL, C. (2000). Educación, territorio y responsabilidad ciudadana, en J.A. Garde (Ed.), Informe 2000. Políticas sociales y estado de bienestar en España, 165-187. Madrid: FUHEM.

COYNE, R. K. \& COOK, E. P. (2004). Understanding persons within environments: An introduction to ecological counselling, en R. K. Conyne y E.P. Cook (Eds.), Ecological counselling: An innovative approach to conceptualizing personenvironment interaction, 3-35. Alexandria, VA: American Counseling Association.

FERNÁNDEZ-LARRAGUETA, S., FERNÁNDEZ-SIERRA, J. \& RODRIGO, M. (2014). Coordinación interprofesional en los centros educativos: una apuesta para la inclusión. Estudios Sobre Educación, 27, 193-211. 
GARCÍA, R. J., MORENO, J. M. \& TORREGO, J. C. (1993). Orientación y Tutoría en la Educación Secundaria. Madrid: Edelvives.

HAMMER, K., RIPPER, J., \& SCHENK, T. (2014). Guía de orientación profesional coordinada. Manual práctico para una orientación de calidad en el ámbito educativo (B. Echeverría y M. Martínez, Trad.). Barcelona: Fundación Bertelsmann.

HERVÁS AVILÉS, R. Ma . (2006). Orientación e intervención psicopedagógica y procesos de cambio. Granada: Grupo Editorial Universitario.

LEY ORGÁNICA 8/2013, de 9 de diciembre, para la mejora de la calidad educativa. BOE núm. 295 (10 de diciembre de 2013), 97858-97921.

LONGAS, J. \& MOLLA, N. (2007). La escuela orientadora. La acción tutorial desde una perspectiva institucional. Madrid: Editorial Narcea.

MANZANARES MOYA, A. (2013). Sistemas integrados de orientación. Una propuesta para la articulación de estructuras y servicios a nivel local. Revista Española de Orientación y Psicopedagogía, 24(1), 62-77.

MCMAHON, H. G., MASON, E. C., DALUGA-GUENTHER, N. \& RUIZ, A. (2014). An ecological model of professional school counseling. Journal of Counseling \& Development, 92(4), 459-471.

NUÑEZ, H., CRESPO, E., ÚCAR, X., \& BERÑE, A. (2014). Enfoques de evaluación orientados a la participación en los procesos de acción comunitaria. Pedagogía Social, 14,79-103.

RODRÍGUEZ ROMERO, Ma.M. (1996). El asesoramiento en Educación. Granada: Ediciones Aljibe.

ROSELLÓ NADAL, E. (1998). Reflexiones sobre la intervención del trabajador social en el contexto educativo. Alternativas. Cuadernos de Trabajo Social, 6, 233-258.

SAN FABIÁN MAROTO, J. L. (2006). La coordinación docente: condiciones organizativas y compromiso profesional. Participación Educativa, 3, 6-10.

SANTANA VEGA, L.E. (2006). Currículo, educación sociolaboral y modelo colaborativo. Estudios sobre Educación, 11, 63-88.

SERRA, J. (2008). Trabajar con otros. Elementos de nueva configuración de los servicios educativos públicos. Profesorado. Revista de currículum y formación del profesorado, 12, 1-15.

VÉLAZ DE MEDRANO, C. (1998). Orientación e intervención psicopedagógica. Concepto, modelos, programas y evaluación. Málaga: Aljibe.

VÉLAZ DE MEDRANO, C. (2002). Intervención educativa y orientadora para la inclusión social de menores en riesgo. Madrid: UNED.

VÉLAZ-DE-MEDRANO, C. (2008). Formación y profesionalización de los orientadores desde el enfoque de competencias. Educación XX1, 11, 155-181. 
VÉLAZ-DE-MEDRANO, C. (Coord.) (2009). Educación y protección de menores en riesgo. Un enfoque comunitario. Barcelona: Graó.

VÉLAZ-DE-MEDRANO, C. (Dir.), MANZANO-SOTO, N., \& BLANCO-BLANCO, A. (2013a): Los procesos de cambio de las políticas públicas sobre orientación y apoyo a la escuela: evaluación comparada de sistemas vigentes y emergentes. Estudio múltiple de casos en una muestra de Comunidades Autónomas. Madrid: CNIIE-Ministerio de Educación, Cultura y Deporte.

VÉLAZ-DE-MEDRANO (Dir.), MANZANARES, A., CASTELlÓ, M., RODRÍGUEZ, M., ARZA, N., MARTÍN, E., INSAUSTI NUIN, V., FERNÁNDEZ-RASINES, P., \& DEL FRAGO, R. (2013b). Estudio de casos sobre las políticas públicas de orientación educativa en una muestra de seis comunidades autónomas (1990-2010). Castilla-La Mancha, Cataluña, Galicia, Madrid, Navarra y País Vasco. Madrid: Editorial UNED.

WILMORE, E. L. (2002). Principal leadership: Applying the new Educational Leadership Constituent Council (ELCC) Standards. Thousand Oaks, CA: Corwin Press.

\section{Referencias digitales}

COLL, C. (2004). Las comunidades de aprendizaje. Nuevos horizontes para la investigación y la intervención en psicología de la educación. Comunicación presentada en el IV Congreso Internacional de Psicología y Educación, Almería, España, 30 Marzo - 2 Abril de 2004. Recuperado de: http://www.psyed.edu.es/prodGrintie/conf/CC_Almeria_04.pdf (Consultado el 8 de diciembre de 2014).

CONSEJO DE LA UNIÓN EUROPEA (2004). Proyecto de Resolución del Consejo y de los Representantes de los Gobiernos de los Estados miembros, sobre el fortalecimiento de las políticas, sistemas y prácticas en materia de orientación permanente en Europa. Consejo de la Unión Europea n ${ }^{\circ}$ 8448/04 EDUC 89 SOC 179. Recuperado de: http://www.todofp.es/dctm/todofp/legislacion/councilresolution2004es.pdf?documentId $=0901 \mathrm{e} 72 \mathrm{~b} 800 \mathrm{~d} 01 \mathrm{a} 7 \quad$ (Consultado $\quad$ el 9 de diciembre de 2014).

NATIONAL CENTER FOR TRANSFORMING SCHOOL COUNSELING (NCTSC) (2009). The new vision for school counseling. Recuperado de: http://www.edtrust.org/dc/tsc/vision (Consultado el 03 de diciembre de 2014). 


\section{Correspondencia con los autores}

Consuelo VÉLAZ-DE-MEDRANO

Facultad de Educación

Departamento Métodos de Investigación y Diagnóstico en Educación II Universidad Nacional de Educación a Distancia (UNED)

Juan del Rosal, 14

28040 Madrid, España

e-mail: consuelo.velaz@edu.uned.es

Esther LÓPEZ-MARTÍN

Facultad de Educación

Departamento Métodos de Investigación y Diagnóstico en Educación II Universidad Nacional de Educación a Distancia (UNED)

Juan del Rosal, 14

28040 Madrid, España

e-mail: estherlopez@edu.uned.es

Eva EXPÓSITO-CASAS

Facultad de Educación

Departamento Métodos de Investigación y Diagnóstico en Educación II Universidad Nacional de Educación a Distancia (UNED)

Juan del Rosal, 14

28040 Madrid, España

e-mail: evaexpositocasas@edu.uned.es

Ana GONZÁLEZ-BENITO

Facultad de Educación

Departamento Métodos de Investigación y Diagnóstico en Educación II Universidad Nacional de Educación a Distancia (UNED)

Juan del Rosal, 14

28040 Madrid, España

e-mail: amgonzalez@edu.uned.es 